\title{
IMAGE COMPRESSION USING CDF 5/3 LIFT DWT AND SVM
}

\author{
Revathy K.R ${ }^{1}$, Arun Vikas Singh ${ }^{2}$ \\ ${ }^{I}$ M.Tech Student, Department of ECE, TJIT Bangalore, Karnataka, India \\ ${ }^{2}$ Head and Professor, Department of ECE, TJIT Bangalore, Karnataka, India
}

\begin{abstract}
Image compression has become an unavoidable process in multimedia and communication applications .Here this paper present a better method of image compression using based DWT and Support Vector Machines(SVM). The Discrete Wavelet Transform(DWT) used here is 5/3 lift based DWT. After the application of DWT the Support Vector Machine will classify the minimum value wavelet coefficients. The compression can be done by combining Support Vectors(SVs) and corresponding weights, then the coefficients are quantized and encoded. By doing the reverse process the reconstructed image is generated. Finally the results shows that the proposed method provides high quality image with good Peak Signal to Noise Ratio(PSNR) and Compression Ratio(CR).
\end{abstract}

Keywords: Discrete Wavelet Transform(DWT),Support Vector Machine(SVM), PSNR, Compression Ratio(CR)

\section{INTRODUCTION}

Nowadays the storage space is getting reduced because of the increase in multimedia applications. So the image compression is act as an important factor. In common the compressed image can be obtained by removing several redundancies like spatial redundancy, spectral redundancy etc.

There are so many image compression techniques. They are DCT, FFT etc. All transformations gives only frequency domain, where as DWT gives both time and frequency domain.

JPEG 2000 with DWT is the existing image compression technique. One of the advantage of DWT is, by using DWT we can perform both lossless and lossy image compression

In this paper a hybrid technology of DWT and SVM is introduced. SVM is a machine learning algorithm. According to input and output SVM regression can be achieved. This method gives better compression ratio and PSNR value than other techniques. At first the input image undergoes pre-processing. Then apply 2D DWT on the preprocessed image. DWT helps to perform both lossless and lossy image. The DWT used here is a 5/3 lift based DWT. At last the SVM regression can be applied to get better results.

\section{LITERATURE SURVEY}

Saied Fazil et al. [1], introduced a new method of image compression using Support Vector Machine (SVM) and DWT. SVM is the one of the common machine learning algorithm. SVM regression can be achieved by using this method. This technique gives more PSNR value and compression ratio than the existing techniques. All existing techniques based on DCT where as here a 2D DWT is used for image compression.
Jaya Shrivastava et al. [2], proposed a method to perform image compression by using modified discrete wavelet transform and high boost filter. There are so many other techniques to perform image compression, but all these techniques reduces the image quality and along with that some noise will be added. But in this paper a very effective and efficient method is introduced to do image compression by keeping the image quality as it is. Here the image compression can be achieved by removing coding redundancy, inter pixel redundancy and psycho-visual redundancy.

Le Hoang Thai et al. [3], proposed an image classification using Support Vector Machine and artificial neural network. The main goal of image classification is to determine its different categories KNN, Adaboosted, Artificial Neural network, Support Vector Machine are the various techniques for image classification. But except SVM and ANN all other techniques are little complicated and also precision of SVM and ANN are higher than other methods.

Abirami et al. [4], presented an approach for image compressed based Wavelet Support Vector machine kernels. In earlier years JPEG is the most commonly used image compression technique. Then the DCT is replaced by DWT. Because so many drawbacks of DCT are replaced by DWT. The main goal of this paper is to perform the image compression in a better way by using wavelets along with the machine learning algorithm.

Jinal patel et al. [5], introduced an implementation of the 5/3 lifting 2D DWT. The main use of Discrete Wavelet Transform is to divide the still image into sub bands of different frequency components. 2D DWT helps to achieve higher compression ratio. This paper described about DWT and image compression, lifting scheme of DWT, lifting scheme of $5 / 3$ wavelet filter and image compression parameters. 
Pacha Sreenivasulu et al. [6], proposed a novel wavelet approach for near lossless image compression using modified duplicate free run length coding. This is a type of lossless method used as a real time application. In this paper the author has done the comparison between the proposed technique and other existing techniques.

Anand D Darji et al. [7], introduced a hardware architecture for multilevel lifting based discrete wavelet transform. Three hardware architecture namely FMA, PMA and RMA are used to perform DWT. To save area and power a new technique is used in PMA, which is used PMA, which is called as clock gating technique.

\section{PROPOSED METHODOLOGY}

Two important techniques used in proposed methodology are 5/3 lift DWT and Support Vector Machine (SVM).

\subsection{5/3 Lift DWT}

Normally the DWT is used to divide the image into different sub bands. The two-dimensional Discrete Wavelet Transform (2D DWT) is built up as a key operation in image handling. In the region of image compression, the 2D DWT has unmistakably won against its forerunner, the 2D Discrete Cosine Transform.

The lifting scheme consist of 3 steps: Splitting, lifting and scaling. Splitting means the image will split into odd and even components. Lifting itself consists of two steps: prediction and updation. In scaling step the values will be in the form of normalized vectors. In 5/3 lift DWT, 5 is lowpass coefficients and 3 is high-pass filter coefficients.

\subsection{Support Vector Machine (SVM)}

SVM is a machine learning algorithm which plays a vital role in image compression. Normally SVM is used to find minimum number of wavelet coefficients. SVM has great speculation capacity. Regression can accomplish pressure between the input and the output with data dependency.

The thought of Support Vector Machine is to make a hyper plane in the middle of information sets to demonstrate which class it fits in with. The test is to prepare the machine to comprehend structure from information and mapping with the right class mark, for the best result, the hyper plane has the biggest separation to the closest preparing information purposes of any class.

In this technique a $256 \times 256$ image is considered as input image. In the first step the image undergoes pre-processing using Gaussian filter. After pre-processing tiling should be done on the whole image. In the next step we apply a 2D 5/3 lift based DWT.To get minimum number of wavelet coefficients SVM regression is applied. Then the compression is obtained by combining all support vectors and weights. This weight vectors should be quantized and encoded.

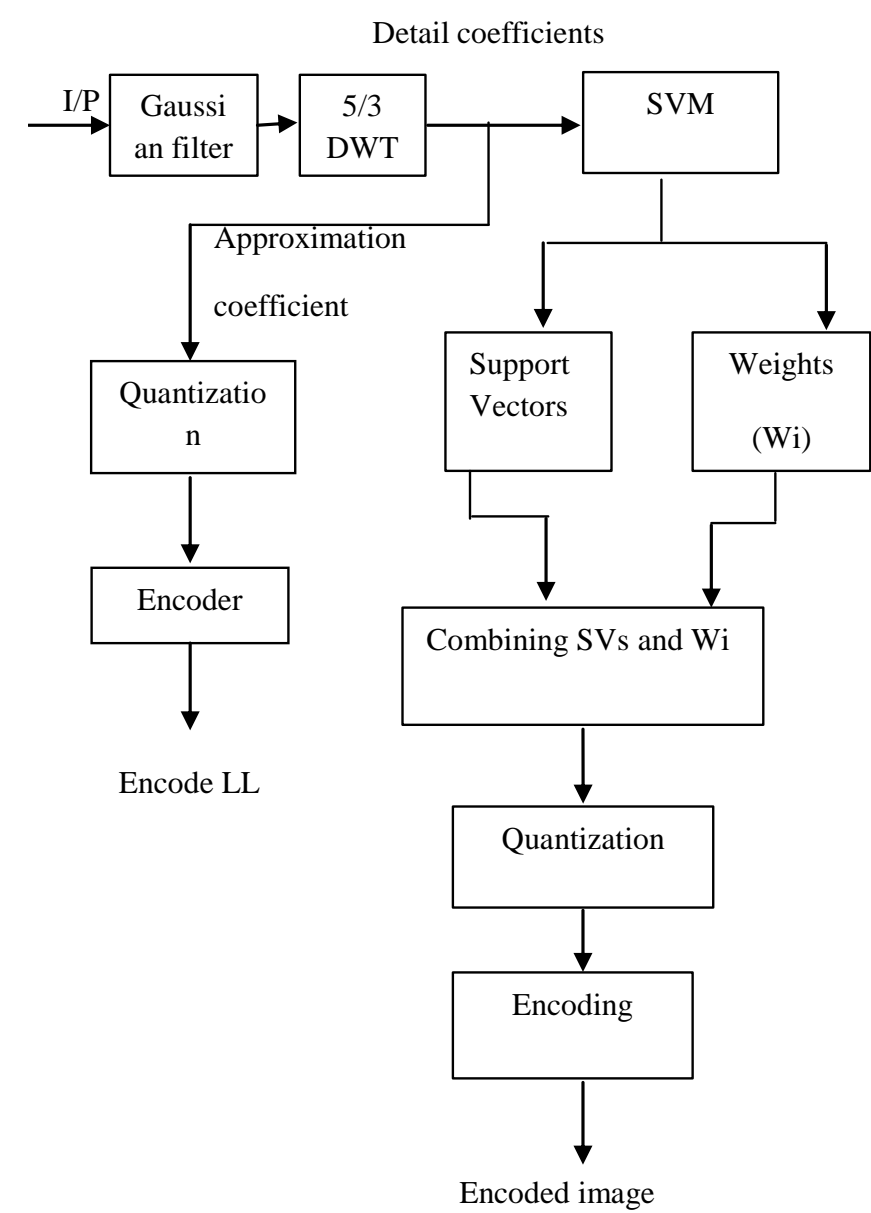

Figure 1: Block diagram of proposed methodology

\subsection{Algorithm}

The algorithm for proposed methodology is given below

Step 1: Take a $256 \times 256$ image as input. Pre-process the input image by using Gaussian filter.

Step 2: Divide the image into small tiles by using raster scan tiling

Step 3: Apply 5/3 lift based DWT on each tile of the image. The image will be divided into approximation and detailed coefficients

Step 4: Apply SVM to find minimum number of wavelet coefficients

Step 5: Find Support Vectors(SVs) and their corresponding weights (Wi) then combine them

Step 6: Perform quantization and encoding by converting decimal to binary

Step 7: To reconstruct the image perform the reverse process

The steps involved in reconstruction of the image is shown in the block diagram 


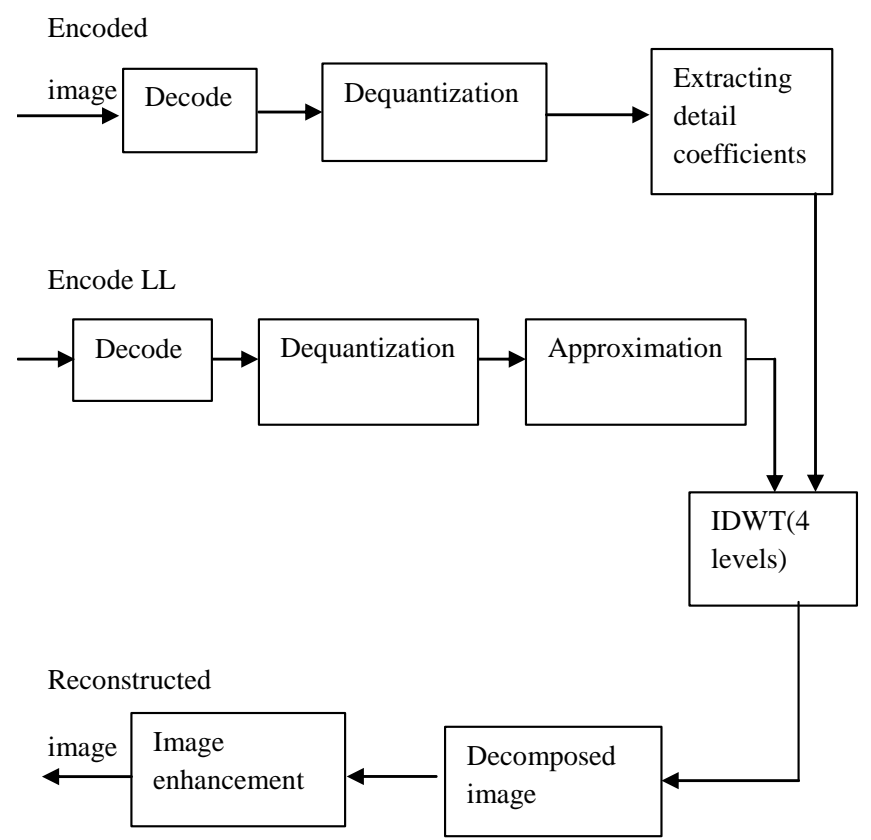

Figure 2: Block diagram of reconstruction

\section{RESULT ANALYSIS}

The different parameters of the output image are PSNR and Compression Ratio (CR). The result images are shown in figure 3

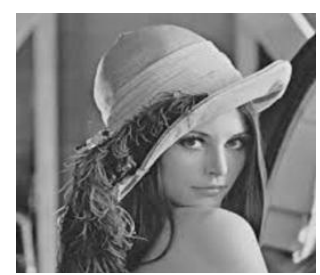

(a) Original image

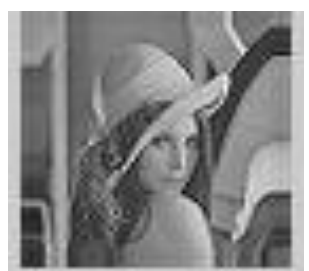

(b) Compressed image

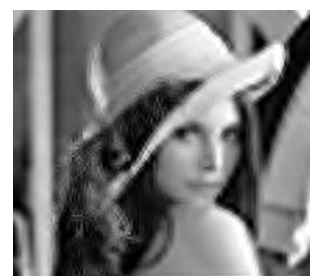

(c) Reconstructed image

Figure 3: Result images

The comparison of different parameters are as shown in table 1

Table 1: Comparison of parameters

\begin{tabular}{|l|l|l|l|l|}
\hline \multirow{2}{*}{ Image } & \multicolumn{2}{|l|}{ Our algorithm } & \multicolumn{2}{l|}{$\begin{array}{l}\text { Existing } \\
\text { algorithm }\end{array}$} \\
\cline { 2 - 5 } & CR & PSNR & CR & PSNR \\
\hline Cameraman & 44.529 & 14.825 & 34.65 & 20.28 \\
\hline Lena & 43.759 & 19.827 & 33.39 & 18.28 \\
\hline Mandrill & 45.873 & 16.792 & 29.84 & 18.46 \\
\hline
\end{tabular}

\section{CONCLUSION}

Table 1 shows comparison of parameters of different images. In this paper a new image compression technique has been introduced. This hybrid technique provides high compression ratio than existing algorithms.

\section{REFERENCES}

[1] Saied Fazli, Siroos, Toofan and Zahra Meehrana,"JPEG Image Compression Using SVM and DWT", International Journal of Science and Engineering Investigations, Vol. 1, Issue 3, pp 53-57, April 2012

[2] Jaya Shrivastava and Neelesh shrivastava, "Image Compression Using Modified Discrete Wavelet Transform With Spatial Domain High Boost Filter", International Journal of Electrical and Computer Engineering, Vol. 1, Issue 4, pp 108-113, April 2015

[3] Le Houng Thai, Tran Son Hai and Nguyen Thanh Thuy, "Image Classification Using Support Vector Machine and Artificial Neural Networks', International Journal of Information Technology and Computer Science, Issue 5, pp 32-38, May 2012

[4] Abirami J, Sivasankari S, Narashiman K, "Image Compression Based on Wavelet Support Vector Machine Kernels”, International Journal of Engineering and Technology, Vol. 5, Issue 2, pp 1584-1588, May 2013

[5] Jinal Patel and Ketki Pathak, "Implementation of the 5/3 Lifting 2D Discrete Wavelet Transform", International Journal of Engineering Development and Research, Vol. 2, Issue 3, pp 2953-2957, 2014

[6] Pacha sreenivasulu and Kancharla Anitha Sheela, "A Novel Wavelet Approach for Near Lossless Image Compression Using Modified Duplicate Free Run Length Coding", Journal of Theoritical and Applied Computer Science, Vol. 8, Issue, pp 51-57, 2014

[7] Anand D Darji, Shailendra singh kushwah, Sabbir N Merchant and Arun N Chandorkar, "High Performance Hardware Architecture for Multilevel Lifting Based Discrete Wavelet Transform", EURASIP Journal on Image and Video Processing, pp 1-19, 2014

\section{BIOGRAPHIES}

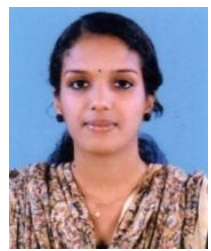

Ms. Revathy K.R is pursuing M. Tech degree in Digital Communication and Networking from $T$. John Institute of Technology, Bangalore affiliated to Visvesvaraya Technological University.

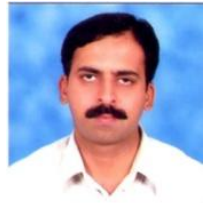

Dr. Arun Vikas Singh is the Head of the Department of Electronics and Communication Engineering, T. John Institute of Technology, Bangalore. He has completed his doctorate in image processing. 